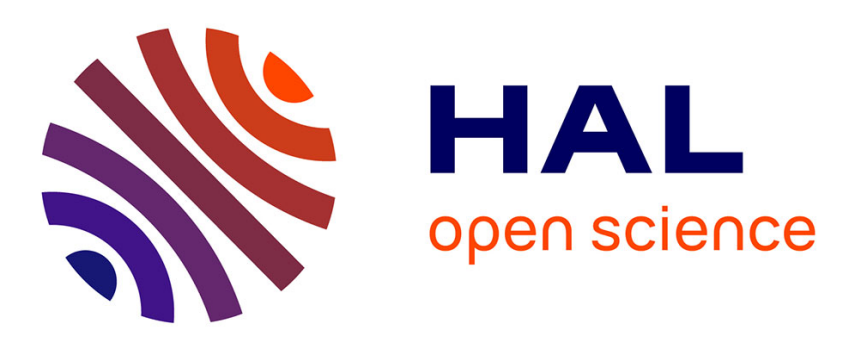

\title{
THE ABSOLUTE DETECTION EFFICIENCY OF A CHANNELPLATE ELECTRON MULTIPLIER
}

\author{
T. Hashizume, T. Sakurai
}

\section{To cite this version:}

T. Hashizume, T. Sakurai. THE ABSOLUTE DETECTION EFFICIENCY OF A CHANNELPLATE ELECTRON MULTIPLIER. Journal de Physique Colloques, 1986, 47 (C2), pp.C2-425-C2-430. 10.1051/jphyscol:1986265 . jpa-00225699

\section{HAL Id: jpa-00225699 https://hal.science/jpa-00225699}

Submitted on 1 Jan 1986

HAL is a multi-disciplinary open access archive for the deposit and dissemination of scientific research documents, whether they are published or not. The documents may come from teaching and research institutions in France or abroad, or from public or private research centers.
L'archive ouverte pluridisciplinaire HAL, est destinée au dépôt et à la diffusion de documents scientifiques de niveau recherche, publiés ou non, émanant des établissements d'enseignement et de recherche français ou étrangers, des laboratoires publics ou privés. 
JOURNAL DE PHYSIQUE

Colloque $C 2$, supplément au $n^{\circ} 3$, Tome 47 , mars 1986

page $\mathrm{c} 2-425$

THE ABSOLUTE DETECTION EFFICIENCY OF A CHANNELPLATE ELECTRON MULTIPLIER

\author{
T. HASHIZUME and T. SAKURAI
}

The Institute for Solid State Physics, The University of Tokyo. Roppongi, Minato-ku, Tokyo 106, Japan

\begin{abstract}
Making use of the high-performance focusing-type ToF atom-probe, we have examined detection efficiency of a micro-channelplate on an absolute scale. The detection efficiency for single ion impacts is approximately $60 \%$, close to the fraction of the active channel area of the surface. It may increase slightly, but no more than a few percent contrary to Panitz's clame, even the front surface is positively biased.
\end{abstract}

\title{
Introduction
}

For last decade or two, micro-channelplate electron multipliers (channelplates) have been used more and more frequently in various fields of sclences and engineerings for detecting charged particles (ions and electrons) and photons. The detection efficiency is of primary importance and has been lnvestigated as a function, of incident beam's energy and angle as well as chemical species in the case

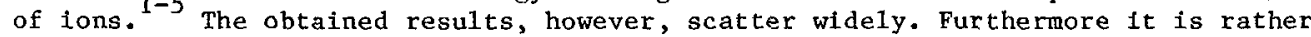
difficult to measure an absolute detection efficiency, particularly in the case of single ion impacts.

Although it has been generally assumed that the maximum detection efficiency of the channelplate is approximately equal to the value corresponding to the fraction of the active surface area occupied by the channels and may not increase much, some people speculated that it may increase significantly under favorable conditions. Panitz clamed in the last year's FE Symposium that the detection efficiency of a channelplate manufactured by Galileo Electro-0ptics (GEO) can reach close to $100 \%$ when its front surface is biased at approximately 20 yolts and that this information has been made available in a mannual supplied by GEO.

In this paper, making yse of our focusing-type time-of-flight atom-probe with a $100 \%$ detection efficlency, we report that precise determination of an absolute detection efficiency of a channelplate can be made in the case of an ion beam, where this value is most sought after. We have found that it never exceeds the fraction of the active area by more than $4 \%$, regardless of the positive voltage applied to the front surface of the channelplate contrary to the clame by Panitz.

\section{Technique}

The atom-probe we employed in this study has a unique capability of focusing the ion beam, which field-evaporated from the emitter surface and entered into the probe-hole, into a tiny spot of $1 \mathrm{~mm}$ diameter at the detector position after the 
flight path of $3000 \mathrm{~mm}$ (Fig. 1). This focusing power enables us to adapt a channeltron ( electron multiplier) which attains $100 \%$ detection efficiency to collect and detect all the incoming ions. This superb performance of the $100 \%$ detection efficiency of the atom-probe has been demonstrated using various specimen emitters, including $\mathrm{W}$, Mo, Mo-0.3at\%Re ${ }^{9}$ and $\mathrm{Ni}-\mathrm{Cu}$ alloys.

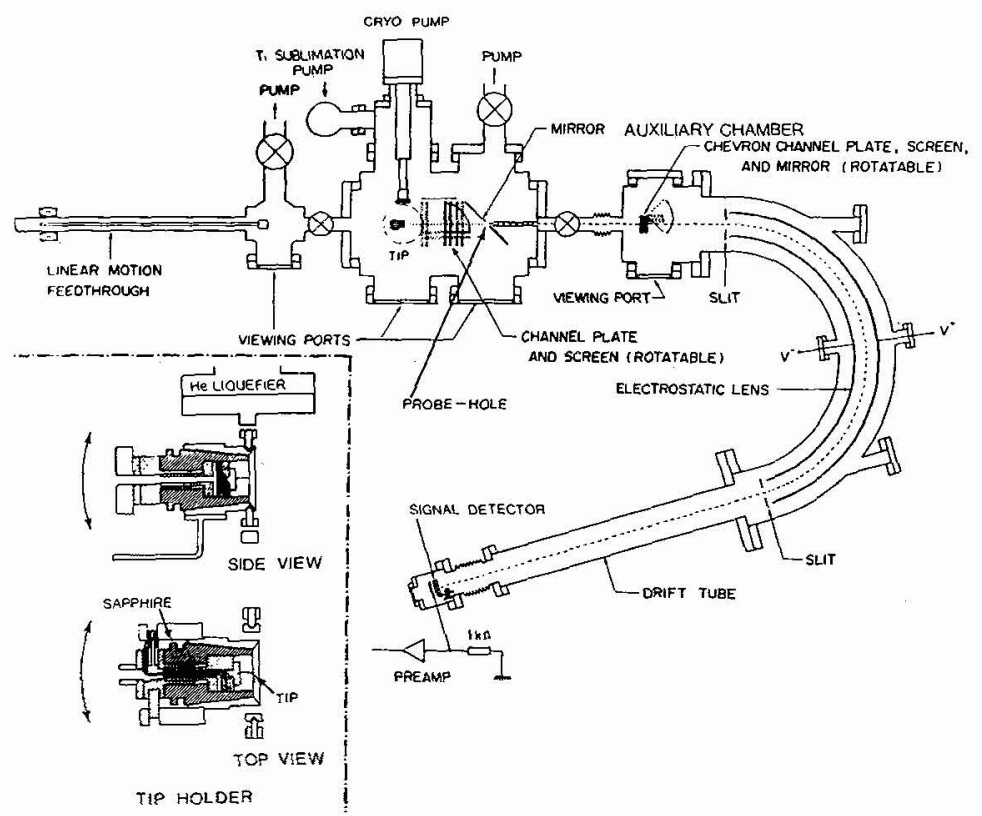

Fig. 1. Schematic of the focusing-type ToF atom-probe. The 2" chevron channelplate at the auxiliary chamber ( $B$ ), newly introduced in front of the ToF section, enables one to view the probing area without ambiguity during atom-probe analysis. And this also helpes one obtain the best alignment of ion beam, essential for the superb focusing of the ion beam.

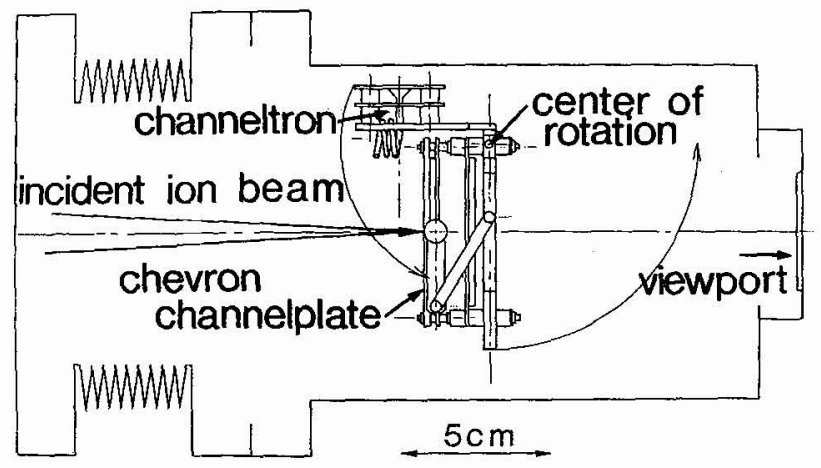

Fig. 2. Schematic of the arrangement of channelplate and channeltron detectors mounted on a single station. Either one can be placed at the position of the atom-probe signal with simple flip-flop motion and can be used for signal detection. This enables us to test and compare the performance of two detectors under exact1y the same conditions.

The method used here is a well-known technique of layer-by-1ayer field evaporation. When the well-defined surface plane, such as the (110) plane, is placed over the probe-hole and pulsed-field evaporation is administered, cumulative $7^{\text {number }}$ of the detected ions against cumulative number of evaporation trigger pulses displays a ladder-shape structure.

The step height corresponds to the total number of signals detected from the 
Individual single layer projected over the probe-hole. Our channeltron detector which detects all the incoming signals can be used as an ideal monitor of ion beam intensity, $\mathrm{N}_{0}$, where $\mathrm{N}_{0}$ is defined as the number of detected ions/layer covered by the probe-hole.

An absolute detection efficiency of other types of signal detectors, channelplates in the present case, can be readily obtained by dividing the beam intensity measured by the specific detector, $N$, from the same surface under the same condition, by that of channeltron, $\mathrm{N}_{0}(\mathrm{Fig} \cdot 2)$.

Result I: Detection effisienency of a channeltron

We demonstrate the $100 \%$ detection efficiency of our focusing-type ToF atom-probe using Mo-Re alloys.

It has been known for some time that an impurity atom on the emitter surface may appear brighter than the rest In FIM. The solute Re atom in Mo-Re alloys may be such a case. The bright atoms randomly dispersed in the Mo FI image were assumed to be Re atoms sitting on the surface, based on the close correlation between the number density of the bright spots and the bulk Re concentrations of various Mo-Re

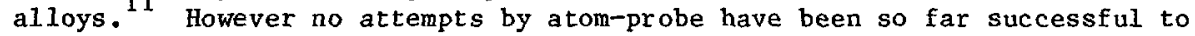
positively identify such random bright atoms as Re, phobąbly because of the insufficient detection efficiency of the instrument.

We have succeeded in positive identification of Re atoms, using our newly built focusing-type ToF atom-probe. The unique festures of this instrument closely related in this work are: (1) The addition of the 2 " chevron channelplate-screen assembly (Fig. 1. (B)) in front of the ToF section, which helpes us achieve precise alignment of ToF ion optics with respect to the emitter. A large Poschenrieder focusing lens together with this optimum alignment has enabled us to achieve a good focusing of the ion beam into a spot of $1 \mathrm{~mm}$ diameter and (2) Use of a channeltron as an atom-probe signal detector (Fig. 2) instead of a conventional channelplate. This is essential for us to achleve $100 \%$ detectability of the incoming ions.

The sample used in this work is a Mo-0.3at\%Re alloy, and was chosen because this level of Re impurity enables us to achieve the optimum condition in FIM operation as to the number density of bright spots and quality of FI image.

The atom-probe analysis of the bulk of this alloy showed that the Re level is $0.33+0.08 a t \%$, in good agreement with the nominal value obtained by chemical analysis. The pulsed field evaporation was carried out at tip temperature of $60 \mathrm{~K}$, in the presence of $10^{-4} \mathrm{~Pa}$ of the He and $20 \% \mathrm{Ne}$ gas mixture, using the pulse ratio of 0.12 . Under this condition, the surface image can be clearly observed during fleld evaporation experiment using both the 5 " single channelplate at the main FIM chamber and the ${ }^{2 "}$ chevron channelplate at the aux1liary chąpber. Mo atoms are detected as $\mathrm{Mo}^{2+}, \mathrm{Mo}^{2+}$ and $\mathrm{Mo}^{4+}$ and $\mathrm{Re}^{\prime} \mathrm{s}$ are detected as $\mathrm{Re}^{3+}$ ions and all the ions are clearly separated in mass histogram.

Individual bright spots in the (110) plane have been analysed in the following ways :

(1) A bright spot appeared in the (110) plane during slow pulse field-evaporation was placed at the center of probe-hole for mass analysis.

(2) Its exact position within a probe-hole was examined, adjusted precisely, and recorded photographically using the chevron channelplate-screen assembly at the auxiliary chamber.

(3) Field evaporation pulses were administered until a signal was detected by the atom-probe channeltron detecter. The image within the probe-hole was examined using the 2" chevron channelplate at the auxiliary chamber every 5 trigger pulses if no signal was registered during those 5 pulses to make sure that the bright spot still remain at the original position.

(4) When the signal is obtained, the surface was examined again to make sure that the signal we obtained corresponds to the bright spot being aimed.

This was continued until the bright spot disappeared and then next bright spot was examined. With this alloy, the bright spot on the (110) plane reappeares by evaporating the surface by approximately every five to ten layers. Since these bright spots on the (110) plane are exposed to the slightly higher field than the substrate atoms, they can be field evaporated easily and in a well-controlled fashion. Indeed these bright spots did evaporate within first several pulses keeping the rest of the surface intact.

A typical result is shown in Fig. 3. Using one particular sample, 27 bright 
spots appeared on the (110) plane by evaporating over 300 layers were examined and a11 of them were detected and identifled as Re ions except one. In F1g. 3, open circles ( 0 ) represent the successful identification of the bright spots as $\mathrm{Re}$ atoms located at various positions within the (110) plane. Cross ( $x$ ) indicates the unsuccessful case where the bright spot disappeared during the field evaporation without any signal registeres at the channeltron detector. No signal was detected in this case probably due to (1) exceedingly large energy deficit during the field evaporation or (2) unexpectedly large deviation of the ion trajectory occasionally seen in pulsed field evaporation. This result clearly shows that our focusing-type ToF atom-probe with the channeltron detector can achieve practically $100 \%$ detection efficiency.

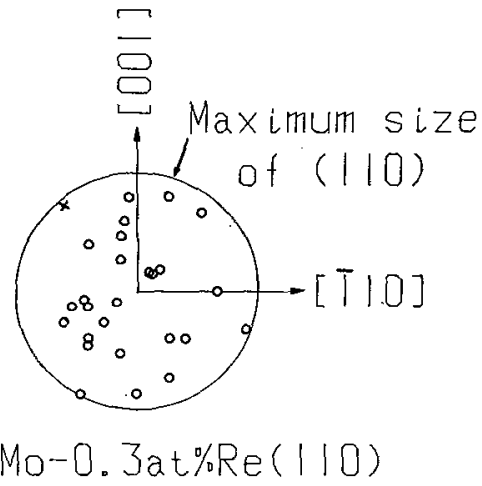

Fig. 3. The position of the bright spots $(0$ and $x$ ) observed on the (110) plane of the Mo-0.3at\%Re tip. Open circles ( 0 ) represent the cases of successful identification of the bright spots by the atom-probe ( all are detected as $\operatorname{Re}^{3+}$ ). Cross $(x)$ represents the unsuccessful case where the bright spot disappeared without detection.

Result II: Detection efficiency of a channelplate

Using a small cone-angle witter with the [110] orientation field evaporation was carried out and the beam intensity measurement was performed after we have established that the surface contour of the emitter remains essentially unchanged by continuous evaporation. The ion beam intensity was measured using both channeltron (GEO Model \#4028) and channelplate (GEO Model \#3040) detectors by placing the probe-hole at the (110) plane and by performing layer-by-layer evaporation in $6 \times 10^{-5}$ $\mathrm{Pa}$ He gas in the range of evaporation voltage of $10.39 \mathrm{KV}$ to $10.69 \mathrm{KV}$ ( Fig. 4). We note that simple flip-flop motion bring either channelplate or channeltron to the detector position without changing any other experimental conditions. Thus the performance of both detectors can be compared on an absolute scale. The beam intensity was determined by averaging the signals over 20 to 30 layers, because of some fluctuation of the measured beam intensity from one layer to another.

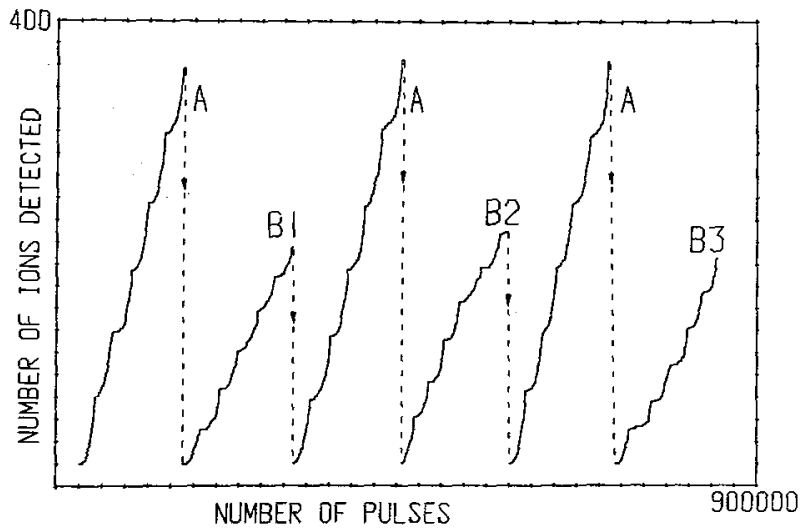

Fig. 4. Cumulative number of detected $W$ lons is plotted against cumulative number of evaporation trigger pulses, alternatively using channeltron (A.) and channelplate (B) detectors. Layer-by-layer evaporation is evident in this figure. In the case of a channelplate detector, the front potentials in volts are : B1, 0 ; $\mathrm{B} 2,98$; and $\mathrm{B} 3,50$.

The result is shown in FIg. 5. Due to the favorable emitter shape having essentially a constant radius, the beam intensity ( Ions / layer) was found to remain constant throughout this experiment which is evident in this figure. The 
channeltron was operated in the pulse counting mode ( or saturation mode) and has yielded $\mathrm{N}_{0}=61.1 \pm 6.0$ lons / layer. The operating condition for the channelplate was varied changing the front surface potential $V_{f}$ from $-1.6 \mathrm{kV}$ to $1.3 \mathrm{kV}$ to test Panitz's clame. However the measured intensity did not change more than $4 \%$ and is $34.8 \pm 5.0$ ions / layer. Fig. 6 shows explicitly the effect of the potential applied to the front surface to the detection efficiency.
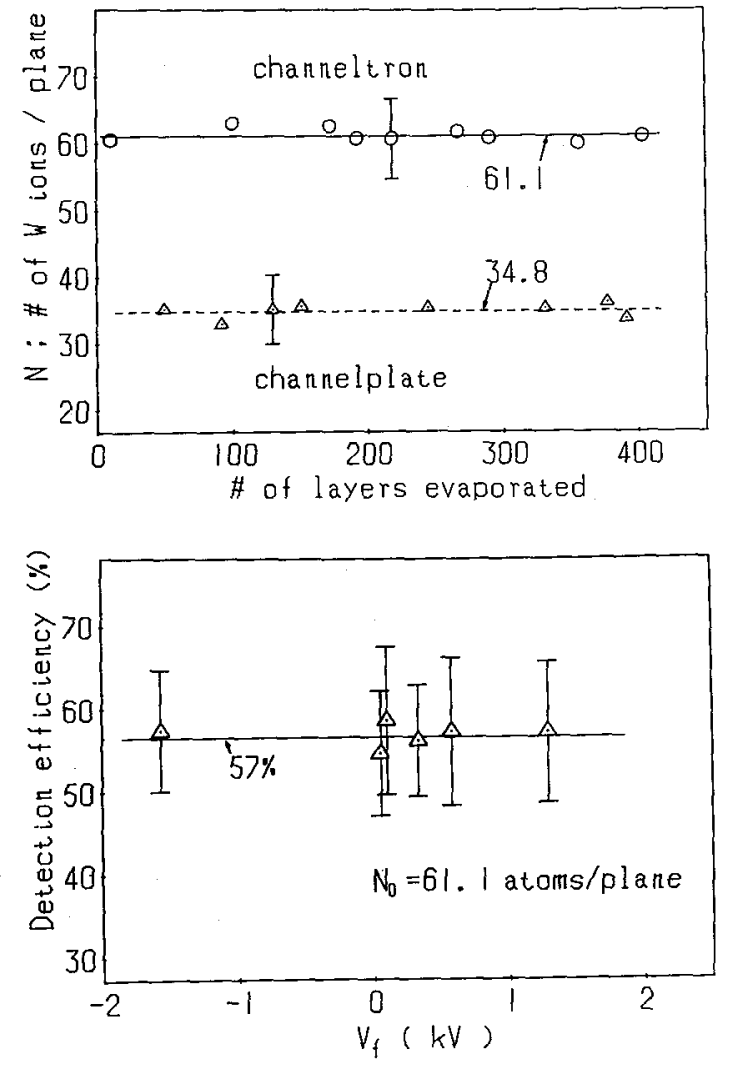

Fig. 5. W ion beam intensity ( ions / plane ) measured during layer-by-layer field evaporation over 400 layers is plotted for both channelplate and channeltron detectors.

Fig. 6 The detection efficlency of a channelplate is measured as a function of the bias voltage applied to the front surface of the channelplate. We do not notice any significant increase in the detection efficiency.

\section{Discussion}

Panitz argued that a small positive potential $\mathrm{V}_{\mathrm{f}}$ applied to the front surface of the channelplate effectively attracts and collects the secondary electrons produced by ions impact between channels and thus may increase the detection efficiency close to $100 \%{ }^{\circ}$ We have observed no such significant improvement in the detection efficiency though the datg point at $V_{f}=98 \mathrm{~V}$ does lie slightly ( $4 \%$ ) above the other values. Gao et al. ${ }^{4}$ also reports that application of a positive potential of approximately $20 \mathrm{~V}$ to the front surface increases the relative
detection efficiency, but only by $20 \%$, in the case of a medium intensity beam ( $10^{3}$ $-10^{4}$ ions $\left./ \mathrm{sec} / \mathrm{cm}^{2}\right)$. The reason for our observation is simple and straightforward. It is indeed possible to collect some $(260 \%)$ of the secondary electrons emitted outward at the front surface of the channelplate by applying a positive potential. However the energy of those secondary electrons is in the range of 3 to $5 \mathrm{eV}$ and the detection efficiengy $y_{5}$ of those low energy electrons is extremely small as is reported by many authors. $1,2,5$ This is why the gain of a channeltron electron multiplier is a strong function of the voltage applied between two ends of a channel.

We have examined only two channelplates supplied by Galileo Electro-optics with different bias angles. However the present conclusion can be readily generalized. other channelplates by different manufactures should show essentially the same 
results since the basic feature that the portion of the active channel area is in the range of $60 \%$ of the total surface area do not vary from one channelplate to another. The bias angle and semiconductor coating of the channel affect only the gain but not the efficiency.

\section{Conclusion}

We report the measurement of the detection efficiency of a channelplate multiplier in the case of single ion impacts without any ambiguity. The absolute detection effictency of the channelplate is determined to be $57 \pm 8 \%$ in this study. This value is very close to the fraction of the active channel area of the front channelplate surface and also agrees well with the independent measurement by Gao et al. using a medium intensity ion beam.

This implies that a channelplate is rather a poor choice of the detector when used in atom-probe FIM's by which ope often tries to analyze individual atoms of

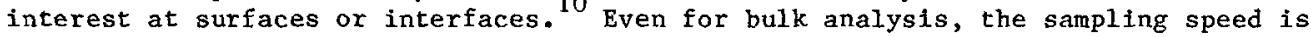
reduced by as much as $40 \%$ using a channelplate detector.

\section{References}

1. See "Review of the influence of radiations on channeltrons and channelplates" by J. P. Macau, J. Jamar \& S. Gardior, IEEE Trans. Nuc1. Sci. 23, 2049 (1976).

2. M. Galanti, R. Gott \& J. F. Renaud, Rev. Sci. Instrum. 42, 1818 (1971).

3. R. E. Kennerly, Rev. Sci. Instrum. 48, 1682 (1982).

4. R. S. Gao, P. S. Gibner, J. H. Newman, K. A. Smith \& R. F. Stebbings, Rev. Sci. Instrum. 55, 1756 (1984).

5. R. R. Garuganthu \& W. G. Wilson, Rev. Sci. Instrum. 55, 2030 (1984).

6. J. A. Panitz \& J. A. Foesch, Rev. Sci. Instrum. 47, 44 (1976).

7. T. Sakurai, T. Hashizume \& A. Jimbo, Appl. Phys. Lett. 44, 38 (1984).

8. T. Sakurai, T. Hashizume; A. Jimbo \& A. Sakai, unpublished.

9. T. Sakurai, T. Hashizume \& A. Jimbo, J. Physique Co1loq. 45, C9-343(1984).

10. T. Hashizume, A. Jimbo \& T. Sakurai, J. Vac. Sci. Technol. A3, May-June issue (1985) in press.

11. K. Teramoto, H. Morikawa, Y. Yashiro, N. Igata \& A. Kohyama, Abstract of the 25th International Field Emission Symposium, p44, Albuquerque, N. M. USA, Aug. 1987.

12. S. V. Krishnaswarmy, S. B. McLane \& E. W. Müller, Rev. Sci. Instrum. 46, 1237 (1975).

13. T. Sakura1, G. Marlow \& Y. Kuk, Japan. J. App1. Phys. 19, L167 (1980). 